Editorial: Volume 3, Issue 2, 2012

\title{
Methodological developments
}

\section{Sophia Psarra}

Reader of Architecture and Spatial Design

The Bartlett School of Graduate Studies, UCL

Pages: $x x i i-x x v$

\section{editorial}

The Journal of Space Syntax

ISSN: 2044-7507 | Year: 2012 | volume: 3 | issue: 2 | Online Publication Date: 28 December 2012 


\section{Editorial: Volume 3, Issue 2, 2012}

\section{O Methodological developments}

The ways in which we understand buildings and cities in space syntax research is inextricably linked with the development of methods, tools and the innovative use of technology. The Autumn/Winter 2012 Issue of JOSS is dedicated to methodological developments, presenting two papers that focus on space syntax measures, and a third one that explores video displays and hybrid spatial topologies.

Space syntax is work in progress, continuously evolving through theoretical and methodological challenges that demand new ways of conceptualising, representing, measuring and evaluating space and use patterns. This Issue emerges from the need to focus the debate on the role of technology and analytical innovation in the development of our field so as to encourage and facilitate the development of innovative methodological practice. The papers presented here draw attention: firstly, to advanced theoretical and analytical ideas currently under development that are propelled by new measures (Hillier, Young and Turner, 2012, p.155-193); secondly, to older measures and analytical procedures that are being revisited, and their potential to lead to new knowledge (Krüger and Vieira, p.194-203); and finally, to how technologies of communication and interaction affect the ways in which we use space and develop social relationships (Schnädelbach 2012, p.204-222).

Starting with new methodological challenges, we are pleased to present a paper by Bill Hillier, Tao Young and Alasdair Turner ('Normalising least angle choice in Depthmap - and how it opens up new perspectives on the global and local analysis of city space', p.155-193), which is an extension of Hillier's keynote presentation at the $8^{\text {th }}$ Space Syntax International Symposium at the Pontificia Universidad Católica of Chile in January 2012 ${ }^{1}$. The paper presents a method for normalising the measures of 'integration' ('mathematical closeness') and 'choice' ('mathematical betweeness') for angular distance, through a comparative analysis of 50 urban systems of different sizes which is enabled by the two measures. The paper also puts forward new theoretical insights into cities and their global structuring. As the authors explain, the research arose due to the paradox of complex segregated designs producing overall higher rates of movement than integrated ones. Using justified graphs to demonstrate why this is the case, Hillier, Young and Turner show that the more the system is segregated, the more choice it generates. The solution to this paradox is provided by the two normalised measures, termed $\mathrm{NACH}$ (normalised angular choice) and NAIN (normalised angular integration). Both measures are tested against size, movement rates, and different radii, with all tests showing that they are independent of the size of urban systems, provide slightly better predictions of movement than choice and integration, and normalise across a range of radii.

Using a technique of a four-pointed star, the authors employ the two measures in a comparative analysis of 50 cities. Starting with American cities such as Manhattan, Chicago, Denver, Las Vegas, Atlanta and New Orleans, they move on to compare European cities such as London to Far-Eastern cities such as Tokyo and Kyoto, and Near-Eastern cities such as Hamedan and Shiraz. The significance of normalised measures is not simply in the capacity to compare cities, such as Manhattan Island with 6296 segments, with the whole city of Chicago with $136,988$ segments ( $p .171)$, and rank them in terms of the strengths of their foreground and background networks, but also to index the degree of their global structuring. The final part of the paper describes the
Notes:

1 'Advancing Depthmap to Advance our Understanding of Cities: Comparing Streets and Cities and Streets to Cities 
global structure of 12 cities' intertwining configurational values (NACH) with an ideal axial geometry that consists of radial, lateral and ring systems. Putting numbers and geometry together, the authors show that Manhattan, Beijing and Kyoto are different instances of a geometric grid. Manhattan is democratically and economically driven, while Beijing and Kyoto are about the spatialisation of a top-down social order. Athens and Santiago on the other hand, are paradigmatic cases of the way in which the geometry of the background grid drives the evolution of cities at the level of the area. Finally, like Manhattan, London and Tokyo are economically driven, but unlike Manhattan have grown linearly rather than laterally, through strong foreground grids followed by the background network that fills the interstitial areas. The last stages of the discussion cast light onto the long-standing division between geometric and organic cities, suggesting that geometrical order and organic growth are not opposite notions but form a continuum. Geometry is applied to the growing city 'somewhere between the whole city and the line' (p.187), the foreground or the background network, and for reasons that are related to economic or social factors.

'Normalising least angle choice in Depthmap' marks a significant step in space syntax research, allowing a numerical comparison between cities of diverse size and context. In addition to this gain, the authors provide new and detailed ways of understanding the local and global structuring of cities. A dual definition of global structure is adopted - configurational and geometrical - showing that cities can give similar or different configurational spatialisation to a short range of geometrical systems and their combinations (radial, lateral and ringy). Recognising the embeddedness of geometry in the configurational network, the authors show that the way in which deliberate thought is applied to the evolution of cities is not through simplistic descriptions that single out the individual line or focus on the city as a whole, but through the way in which geometry shapes the foreground and background network over time. Beautifully illustrated with maps that highlight elements of the foreground grids in 12 cities, 'Normalising least angle choice' opens up a large field of research possibilities. The dual definition of structure using configuration and geometry brings to the fore both notions in understanding cities as self-organising entities. Hillier, Young and Turner's new measures not only enable the understanding of self-organisation through synchronic analysis, but can also allow researchers to perform a comparative analysis of the various stages in the evolution of cities through space-time descriptions, so as to reconstruct a retrospective sequence of spatial morphology and self-organisation.

Following the normalisation of values in segments maps offered by Hillier, Young and Turner, Krüger and Vieira's contribution to this Issue - 'Scaling relative asymmetry in space syntax analysis' - takes us back to the early years of the development of analytic measures in space syntax. The authors begin by reviewing a paper by Krüger - 'On node and axial maps: distance measures and related topics' (Krüger, 1989) - and move on to present research on the measure of centrality (1/ $\mathrm{RA}$ ) with a dispersion measure in order to obtain a more complete picture of the distribution of depth in axial maps. Their proposition is that in order to compare axial maps of different sizes one should have the same yardstick, not only in terms of their mean depth, but also in terms of the dispersion of values. Their study takes into account the standard deviation of depth from an axial map, and proposes the measure of Scaled Relative Asymmetry (SRAi) of axial maps.

Compared to Hillier, Young and Turner's work, Krüger and Vieira's paper reminds us that when research on certain technical problems has advanced so far, further work proceeding from the older premises seems, at first, a reversal of direc- 
tion. Such reversals, which are often associated with a geographical dispersion of research, create the possibility of erecting a new set of possibilities, additional to or even greater than what has been achieved so far by turning back to older methods of measuring and representation. These reversals lay the ground for a creative reengagement with older problems. So Krüger and Vieira propose that Scaled Relative Asymmetry will provide promising correlations with natural urban movement. They also explain that 'since two-dimensional plane axial maps do not apply to multi-storey buildings, the diamond shape will be mapped onto a sphere in order to take into account the genus of a three-dimensional surface, while novel derivations should be made for the mean depth and the standard deviation of these axial maps' (p.203). It is with particular reference to complex buildings as three-dimensional systems that segment analysis gives way to axial analysis, demonstrating that older tools are still key tools that if creatively re-interpreted can resolve long-standing problems in theory and analysis.

Holger Schnädelbach's paper, 'Hybrid spatial topologies' explores buildings and moves in an entirely new direction to the previous two papers. It investigates the effects of introducing video communication technologies into architecture - extending architectural topology into remote spaces and expanding the opportunities for social interaction. Schnädelbach introduces the concept of the 'spatiotechnological isovist' (an isovist through a space and a video connection) to capture how dynamic camera properties shape architectural visibility to physically and remotely connected spaces. Starting from the acknowledgement that video technologies have become a core part of our lives, he shows 'how technologically shallow regions augmenting physically shallow regions in an architectural space can result in an increase in chance encounters' (p.219) and influence movement patterns. In addition, making connections across close and distant spaces can be more democratically distributed to the users of spaces than these connections being imposed on them by architects or people who make decisions on how spaces are used in organisations. Schnädelbach concludes that hybrid spatial topologies can generate entirely new architectural interfaces and social relationships, encourage informal and accidental encounters, and have a direct impact on social life. These kinds of interfaces are not entirely possible in physical architectural space as this cannot be flexible or malleable.

As Schnädelbach points out, the idea that architecture can be visually and virtually extended is nearly as old as architecture itself. Paintings depicting naturalistic scenes featured in buildings in the Roman and ancient Greek periods, while in the Renaissance and Baroque eras, rich and elaborate frescos stretched interior spaces into illusionary distance. In addition to this, mirrors and reflective surfaces were also in use, challenging perceptions of the material and immaterial, actual and virtual. However, as Schnädelbach shows, the hybrid architectural topologies enabled by technology extend and augment architecture's capability to shape not only our spatial cognition and perception, but also our social life. Holger's spatio-technical isovists challenge us to consider our spatial representations, taking into account the ways in which displays shape and alter visual fields in architecture. As patterns of human activity increasingly involve remote communication environments, video displays, computer simulated spaces in real and imaginary worlds, and computer games among others, our theoretical and analytical capacities should extend from physical spaces to various mixed reality scales. Finally, as is often the case, work that addresses the current state-of-the-art in an area implicitly carries with it issues that remain unresolved from previous stages of development. 'Hybrid spatial topologies' therefore raises questions of visibility versus permeability, the relation between seeing and moving, and 
the ways in which tensions between the two types of properties shape social life in buildings and cities.

With this Issue we begin a new practice and section in the Journal dedicated to short research papers. These can be reports of interesting findings of work in progress, a specific and well-argued theoretical point, or descriptions of empirical studies with original data that confirm theoretical insights. Joy Maina's paper, 'Using space syntax methods to explore abandoned prototype housing units in Tangale land, North-Eastern Nigeria', presented in this Issue falls within this last category. The 'Short Papers' section is also intended to include extended abstracts (1000 to 1500 words with supporting diagrams) or summaries of recently completed PhD theses.

Luiz Amorim's review of Frederico de Holanda's book Exceptional Space (2011, published by FRBH) and Apiradee Kasemsook's review of Rachel Keeton's Rising in the East: Contemporary New Towns in Asia (2011, Amsterdam, NL: SUN Architecture) complete this Issue.

We would like to thank all our authors, reviewers and readers, and send our best wishes for the Christmas break and the New Year to all.

\section{References}

de Holanda, F. (2011), Exceptional space, (kindle edition), FRBH.

Hillier, B., Yang, T. and Turner, A. (2012), 'Normalising least angle choice in Depthmap - and how it opens up new perspectives on the global and local analysis of city space'. In: The Journal of Space Syntax, Vol. 3 (2), p.155-193.

Keeton, R. (2011), Rising in the East: Contemporary New Towns in Asia, Amsterdam, NL: SUN Architecture.

Krüger, M. (1989), 'On node and axial maps: distance measures and related topics'. In: European Conference on the Representation and Management of Urban Change, Cambridge: University of Cambridge.

Krüger, M. and Vieira, A. P. (2012), 'Scaling relative asymmetry in space syntax analysis'. In: The Journal of Space Syntax, Vol. 3 (2), p.194-203.

Maina, J. (2012), 'Using space syntax methods to explore abandoned prototype housing units in Tangale land, North-Eastern Nigeria'. In: The Journal of Space Syntax, Vol. 3 (2), p.223-227.

Schnädelbach, H. (2012), 'Hybrid spatial topologies'. In: The Journal of Space Syntax, Vol. 3 (2), p.204-222.

Sophia Psarra

Editor 